\title{
Results of type II hybrid arch repair with zone 0 stent graft deployment for complex aortic arch pathology
}

\author{
William D. T. Kent, MD, MSc, ${ }^{a}$ Jehangir J. Appoo, MDCM, ${ }^{a}$ Joseph E. Bavaria, MD, ${ }^{b}$ Eric J. Herget, MD, \\ Patrick Moeller, MS, ${ }^{b}$ Alberto Pochettino, MD, ${ }^{d}$ and Jason K. Wong, MD $^{\mathrm{c}}$
}

Objective: To review the early results of a less invasive, single-stage hybrid arch procedure involving replacement of the ascending aorta, arch debranching, and zone 0 antegrade stent graft deployment.

\begin{abstract}
Methods: Between May 2007 and January 2012, 20 patients with both acute and chronic aortic pathology were managed at 2 institutions with a type 2 hybrid arch procedure. Indications included diffuse atherosclerotic aneurysm, false lumen expansion of chronic aortic dissections, penetrating atherosclerotic ulcer, and acute type A dissection. Mean age was $67 \pm 16.8$ years with a mean European System for Cardiac Operative Risk Evaluation II score of $29.5 \pm 19.4$. Postoperative clinical and imaging follow-up was complete to a mean $18.5 \pm 15.3$ months.
\end{abstract}

\begin{abstract}
Results: Successful zone 0 stent graft deployment was achieved in all cases. There was 1 in-hospital mortality $(5 \%)$. A second death occurred at 40 days postoperation. Other complications included a permanent neurologic deficit in 1 patient $(5 \%)$, transient paraplegia in 4 patients $(20 \%)$, and 3 patients had respiratory complications $(15 \%)$. There were no cases of renal failure requiring dialysis. Stent-related complications were identified in 4 patients $(20 \%)$, including 3 type I endoleaks, none of which were at zone 0 . There was 1 type II endoleak and a case of stent infolding. Two patients required a second successful endografting procedure.
\end{abstract}

Conclusions: This single-stage hybrid arch procedure offers an alternative approach to complex diffuse aortic pathology involving the arch. Replacement of the ascending aorta provides a safe location for zone 0 stent graft deployment, eliminating complications of proximal deployment in a native diseased aorta. (J Thorac Cardiovasc Surg 2014;148:2951-5)

When diseases of the thoracic aorta such as dissection, aneurysm, penetrating atherosclerotic ulcer, and pseudoaneurysm involve the arch, total arch replacement is often indicated. In cases of diffuse disease, the conventional approach is a 2-stage elephant trunk procedure requiring a prolonged period of deep hypothermic circulatory arrest for ascending and total arch replacement followed by left thoracotomy for repair of the diseased descending thoracic aorta. Even at centers with expertise in thoracic aortic surgery, this operation is associated with a risk of mortality between $13 \%$ and $36 \%$ in contemporary series. ${ }^{1}$ As an alternative, off-pump hybrid arch debranching techniques with endovascular stent graft

\footnotetext{
From the Division of Cardiac Surgery, ${ }^{\mathrm{a}}$ Libin Cardiovascular Institute of Alberta, University of Calgary, Calgary, Alberta, Canada; Hospital of the University of Pennsylvania, ${ }^{\mathrm{b}}$ Philadelphia, Pa; Division of Interventional Radiology, ${ }^{\mathrm{c}}$ Department of Diagnostic Imaging, Foothills Medical Centre, University of Calgary, Calgary, Alberta, Canada; and Department of Cardiovascular Surgery, ${ }^{\mathrm{d}}$ Mayo Clinic, Rochester, Minn

Disclosures: Jehangir J. Appoo reports consulting fees from Gore Medical. Jason K. Wong reports consulting fees from Cook Medical. All other authors have nothing to disclose with regard to commercial support.

Received for publication April 21, 2013; revisions received June 2, 2014; accepted for publication June 5, 2014; available ahead of print Aug 12, 2014.

Address for reprints: Jehangir J. Appoo, MDCM, Foothills Medical Centre, C828, 1403 29th St, NW, Calgary, Alberta T2N 2T9, Canada (E-mail: jehangir.appoo@ albertahealthservices.ca).

$0022-5223 / \$ 36.00$

Copyright (c) 2014 by The American Association for Thoracic Surgery

http://dx.doi.org/10.1016/j.jtcvs.2014.06.070
}

repair of the arch and descending thoracic aorta have been proposed to eliminate both circulatory arrest and the 2-stage approach. These operations use a debranching graft sewn to the native ascending aorta using a partial occlusion clamp. ${ }^{2,3}$ Although this technique is effective when the ascending aorta is normal, it can be associated with complications when it is not. Type IA endoleak, rupture, pseudoaneurysm formation, and retrograde type A dissection can be the consequence of stent deployment in a diseased, native aorta. ${ }^{4}$ Recently, for diffuse atherosclerotic aneurysms involving the arch, we have used a single-stage hybrid approach without circulatory arrest that involves replacement of the ascending aorta, arch debranching, and antegrade endovascular stent deployment. ${ }^{5}$ This strategy has the advantage of being a single-stage procedure and may reduce the incidence of perioperative neurologic events by eliminating prolonged periods of circulatory arrest. By eliminating deep hypothermia and risks of distal arch replacement, the operation may be more reproducible and result in lower operative mortality. In the longer term, adequate landing zones are present for distal extension if required. Replacement of the ascending aorta with a Dacron graft may theoretically result in a decreased incidence of stent complications, particularly retrograde type A dissection.

Replacement of the ascending aorta with arch debranching and stent graft deployment not only has merit 
for repair of chronic arch pathology, but also for acute diseases. For management of intramural hematoma or type A dissection, the standard contemporary operation involves replacement of the ascending aorta with a beveled hemiarch procedure under a period of hypothermic circulatory arrest usually along with various strategies of cerebral perfusion. Recently, there has been an increasing awareness of late postoperative mortality after acute type A dissection repair due to aneurysm formation, distal malperfusion, or rupture of the residual dissected arch and descending thoracic aorta. To ameliorate this, several groups have suggested total arch replacement with simultaneous stent grafting of the proximal descending thoracic aorta. ${ }^{6,7}$ However, total arch replacement adds significant risk to an operation that already has a $24 \%$ rate of mortality. ${ }^{8}$ Recently, alternative hybrid approaches have been proposed that involve graft replacement of the ascending aorta with arch debranching to facilitate thoracic endovascular repair of the arch and descending thoracic aorta. ${ }^{9-12}$ This less-invasive technique for total arch replacement, when required for acute type A dissection, may decrease bleeding associated with arch replacement under deep hypothermia, decrease the risk of stroke by eliminating the need for circulatory arrest, and treat malperfusion by opening the true lumen distally. Over the long-term, false lumen thrombosis may decrease the incidence of aneurysm formation and rupture.

This series presents the early results of our hybrid arch procedure. Early in this experience it was used for patients with diffuse atherosclerotic aneurysms involving the arch who presented prohibitive risk for conventional surgery. More recently, indications for this operation were extended to acute type A dissection with an indication for total arch replacement. Our goal was to assess the outcome of this operation when performed in both the emergency and elective settings.

\section{METHODS}

From May 2007 until January 2012, 20 patients underwent total arch replacement with a single-stage hybrid arch technique involving replacement of the ascending aorta, arch debranching, and antegrade delivery of an endovascular stent graft. Procedures were performed at 2 centers: The University of Calgary (11 patients) and the University of Pennsylvania ( 9 patients). Initial experience was in an elective setting for patients with an indication for total arch replacement for diffuse aortomegaly but with prohibitive risk for conventional total arch surgery. Comorbidities of cerebrovascular disease, history of cardiac surgery, pulmonary and renal dysfunction, or advanced age meant these patients had unacceptable risk for the conventional approach. Patient demographics and clinical risk factors for this group, managed in the elective setting, are shown in Table 1.

After April 2011 the indications for this hybrid arch technique were expanded to include those with acute type A dissection. Four patients were managed as emergency cases. Indication for hybrid arch surgery in these patients was acute type A dissection with a primary intimal tear or large reentry tear in the arch, a concomitant arch aneurysm, or peripheral malperfusion. Indications for surgery in both the elective and emergency setting are outlined in Table 2.
TABLE 1. Patient characteristics $(\mathrm{N}=20)$

\begin{tabular}{lc}
\hline \multicolumn{1}{c}{ Variable } & Result \\
\hline Mean age, y & $67.05 \pm 16.86$ \\
Mean European System for Cardiac Operative Risk & $29.47 \pm 19.44$ \\
$\quad$ Evaluation II score & \\
Chronic lung disease* & 8 \\
Cerebrovascular disease $\dagger$ & 8 \\
Diabetes & 2 \\
Hypertension & 19 \\
Renal failure $\ddagger$ & 3 \\
Smoker & 8 \\
Previous sternotomy & 6 \\
\hline
\end{tabular}

Values are given as mean \pm standard deviation or $\mathrm{n}$. ${ }^{*}$ Includes chronic obstructive pulmonary disease, asthma, and interstitial lung disease. †Includes stroke, transient ischemic attack, and internal carotid artery stenosis. $\ddagger$ Defined as creatinine $>2.0$ $\mathrm{mg} / \mathrm{dL}$ or dialysis.

Preoperative evaluation was made by multislice computed tomography scans to provide detailed analysis of the entire aortic anatomy to determine size of stent graft; location of distal landing zone; and in the case of dissection, to determine location and extend of dissection flap and evaluate for evidence of malperfusion. Imaging surveillance was performed with computed tomography angiography done before discharge and then at 3 , 6 , and 12 months postoperatively, followed by yearly thereafter.

All patients provided consent preoperatively to the experimental nature of the procedure. The institutional review board at both centers approved postoperative data analysis.

\section{Surgical Technique}

After right axillary cannulation, median sternotomy is performed, and a 2 -stage venous cannula is placed. The patient is heparinized and cardiopulmonary bypass is initiated. The distal ascending aorta is crossclamped in cases of chronic aortic pathology and also for acute type A dissection. The ascending aorta is then resected to the level of the sinotubular junction. In cases of aortic root pathology, resection may include the sinuses and a valve-sparing or composite aortic root replacement may be performed. The ascending aorta is replaced in an end-to-end fashion using a Bavaria graft (Vascutek; Terumo Cardiovascular Systems, Ann Arbor, Mich); a 28-mm prefabricated Dacron tube graft with 4 side-limbs designed for arch debranching (Figure 1). For procedures performed at the University of Pennsylvania and the initial procedures performed at the University of Calgary, a brief period of circulatory arrest with antegrade perfusion was used to perform an open distal anastomosis. Circulatory arrest was not used for the most recent 6 cases. For these, the distal anastomosis was performed with the clamp on. Following completion of the distal anastomosis, arch debranching is performed in a sequential fashion while the patient is still on bypass with the crossclamp off. One limb of the graft is anastomosed to the proximal left subclavian artery or alternatively to the left axillary artery through a separate left infraclavicular incision. The second limb

TABLE 2. Operative indications $(\mathbf{N}=\mathbf{2 0})$

\begin{tabular}{ll}
\hline \multicolumn{1}{c}{ Pathology } & $\mathbf{n}$ \\
\hline Diffuse atherosclerotic aneurysm involving the arch & 9 \\
Penetrating atherosclerotic ulcer with contained rupture localized & 2 \\
to the arch & \\
Arch pseudoaneurysm at proximal extent of existing stent graft & 2 \\
Acute type A aortic dissection with intimal tear involving the arch & 4 \\
Chronic type B dissection with associated aneurysm involving the & 3 \\
arch and descending thoracic aorta & \\
\hline
\end{tabular}




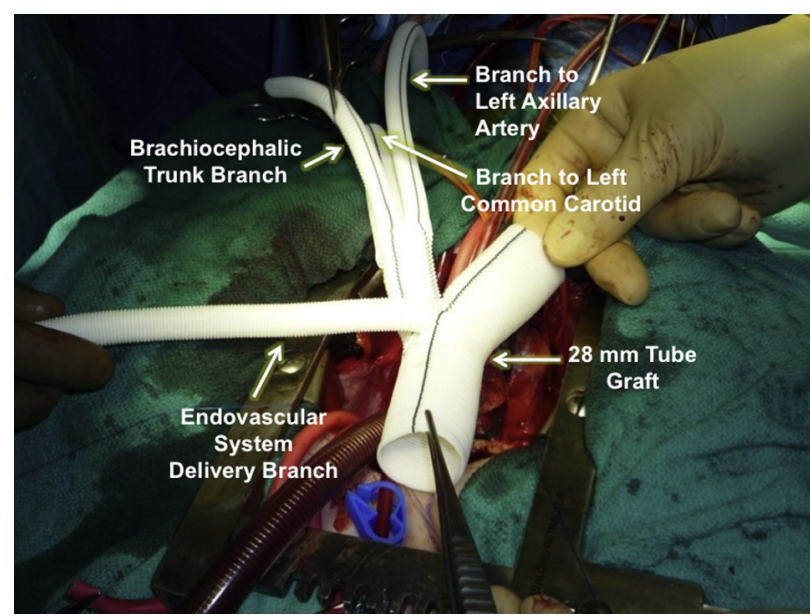

FIGURE 1. Ascending aorta replaced with 4-branch Bavaria graft.

is anastomosed to the left carotid artery and the third, larger diameter graft, to the innominate artery. The patient is then weaned from cardiopulmonary bypass but protamine is not administered. The fourth limb of the Bavaria graft is used for antegrade stent graft delivery (Figure 2). A Gore TAG thoracic endovascular stent graft (Gore TAG Thoracic Endoprosthesis; W.L. Gore and Associates, Inc, Flagstaff, Ariz) is deployed in an antegrade fashion from the ascending aorta to the proximal descending thoracic aorta. The proximal landing zone in the Dacron Bavaria graft is identified on fluoroscopy by a radiolucent umbilical tape fixed to the tube graft immediately distal to the ostium of main side branch (Figure 3). In addition to the hybrid arch procedure, 1 patient had concomitant bypass grafting and 6 patients had concomitant aortic valve repair or replacement. Following successful technical deployment, heparin is reversed, hemostasis is ensured, and the chest is closed.

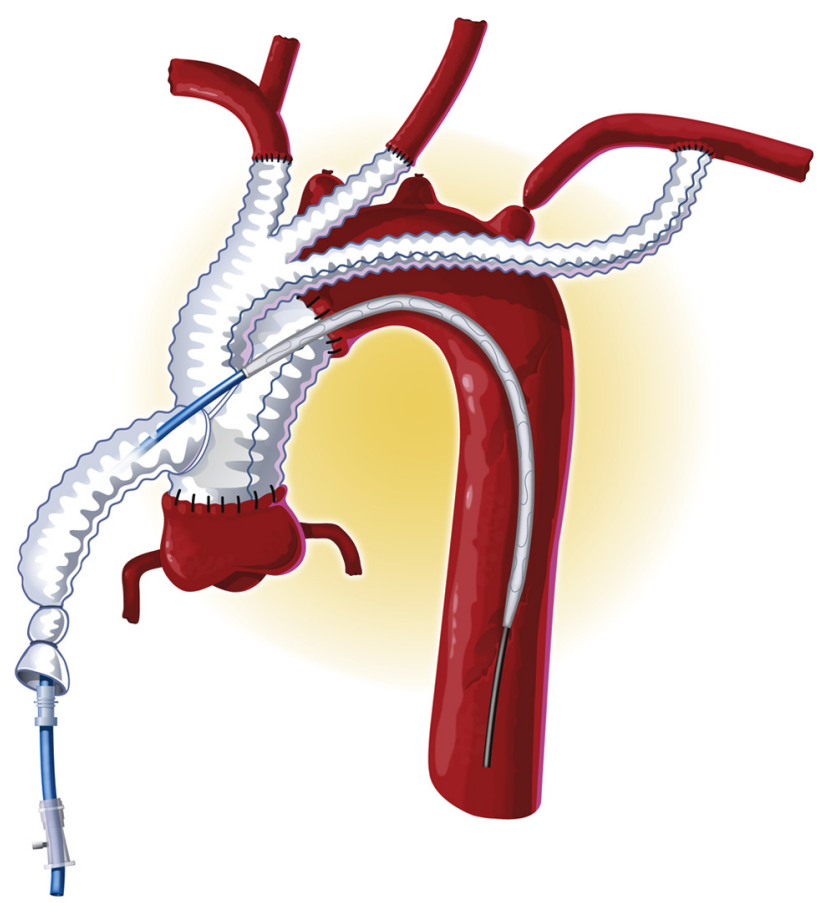

FIGURE 2. Antegrade delivery of endovascular stent graft.

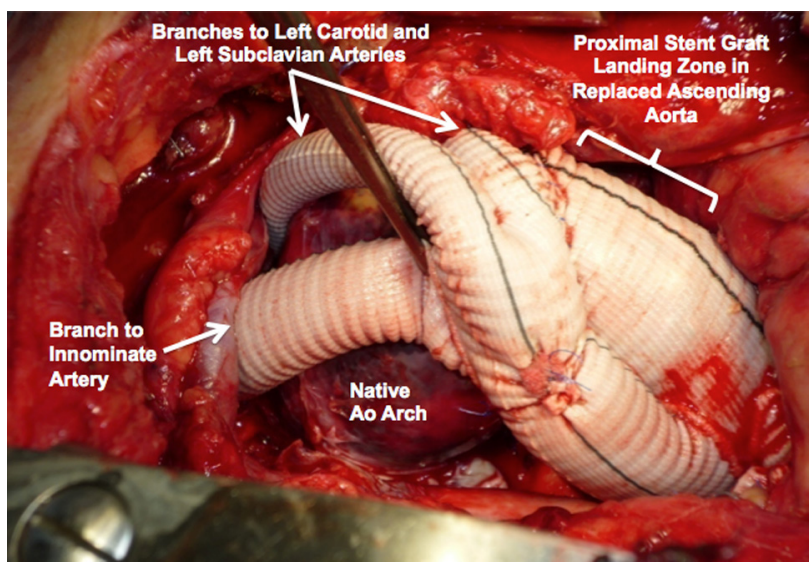

FIGURE 3. Completed arch debranching with stent deployed at zone 0 .

\section{RESULTS}

Patients were followed clinically at an outpatient clinic. Mean duration of follow-up was 563.14 days (range, 411683 days) and was complete for all patients. In the entire series there were 2 deaths. In both cases the operation was performed for acute type A dissection. One was a reoperative case that initially presented as an acute type A dissection and was managed in conventional fashion with replacement of the ascending aorta. Shortly after surgery, the patient was diagnosed with a large pseudoaneurysm involving the arch. A hybrid arch repair was attempted but the patient died intraoperatively. A second death occurred at 40 days postoperatively and was related to a respiratory complication.

Successful stent graft deployment at zone 0 , according to the Ishimaru arch map, was achieved in all cases. Intraoperative details are outlined in Table 3 . In 14 of 20 patients, a period of circulatory arrest was used. Later in the series, circulatory arrest was not performed. Postoperatively, a transient neurologic deficit occurred in 1 patient $(5 \%)$ and there was 1 stroke $(5 \%)$. This patient returned home with a good functional recovery. Paraplegia complicated 4 cases $(20 \%)$, but this was transient lower extremity weakness with complete recovery in all patients. Five patients $(20 \%)$ required reexploration for bleeding in the early postoperative period. Four patients had a prolonged intubation in the intensive care unit, whereas $3(15 \%)$ developed postoperative pneumonia. Despite the intraoperative injection

TABLE 3. Operative data

\begin{tabular}{lcl}
\hline \multicolumn{1}{c}{ Variable } & $\begin{array}{c}\text { Mean } \pm \text { standard } \\
\text { deviation }\end{array}$ & \multicolumn{1}{c}{ Range } \\
\hline Cardiopulmonary bypass, min & $252.40 \pm 58$ & $177-366$ \\
Crossclamp time, min & $95.80 \pm 60$ & $0-213$ \\
Circulatory arrest, ${ }^{*}$ min & $26.70 \pm 27$ & $0-78$ \\
Number of stents placed & $2 \pm 0.92$ & $1-4$ \\
\hline *Circulatory arrest was required in 14 of 20 patients.
\end{tabular}


of contrast, no patients developed renal failure requiring dialysis and no patients required tracheostomy.

Complications related to the endovascular stent graft were identified during the first 30 postoperative days in 3 patients $(14 \%)$. A type I endoleak was identified in all these cases and in 1 patient a concomitant type II endoleak was identified as well. Most cases were recognized intraoperatively and were repaired, whereas only 1 required reintervention with placement of an additional stent through peripheral access. In the 2 other cases, the leak was small and remained stable on serial imaging studies. One patient developed a late infolding at the proximal end of the stent graft. This was successfully managed percutaneously by placing a Palmaz stent to reexpand the stent graft. Imaging follow-up was complete in $100 \%$ of cases with a mean follow-up time of 17.5 months.

\section{DISCUSSION}

Conventional operative management of thoracic aortic pathology involving the arch involves long periods of hypothermic circulatory arrest, which presents high risk for neurologic complications, even with the use of antegrade cerebral perfusion. Recently, to decrease the operative risks and morbidity associated with arch reconstruction, hybrid procedures have been advocated. ${ }^{13,14}$ Most commonly, these are performed off-pump with arch debranching followed by retrograde endovascular stent graft deployment in the ascending aorta to cover the arch and proximal descending thoracic aorta. ${ }^{15-18}$ This offers a less invasive approach and has demonstrated favorable early results. ${ }^{2,19,20}$ However, these procedures require the anastomosis of a debranching graft and the proximal stent graft landing zone to be located in the diseased, aneurysmal ascending aorta. The risks associated with stent graft placement in the ascending aorta and arch are not yet fully understood, but recent reviews have suggested they are substantial. Type Ia endoleak may occur with a frequency of $15 \%$ to $30 \% .^{4,20-22}$ The devastating complication of acute retrograde type A dissection may be as high as $6 \%{ }^{4}$ and is associated with a mortality rate of $42 \%$, according to the European Registry on Endovascular Aortic Repair Complications. ${ }^{23}$ It is assumed that these reported complications have occurred after proximal deployment in relatively normal aortic tissue. Therefore, it is possible that proximal placement in a diseased ascending aorta may result in an even higher rate of these complications. The alternative approach used in our series addresses this issue by replacing the ascending aorta with a Dacron tube graft with 4 side branches. This allows arch debranching and then antegrade stent graft deployment at zone 0 in a robust Dacron tube. As has been previously noted, flat, straight, long, and cylindrical landing zones are ideally suited for stable deployment of stent grafts. ${ }^{22}$ These conditions are met by deployment in the Dacron Bavaria graft and thus the risks of endoleak and retrograde type A dissection may be eliminated.

Addressing concomitant pathology in the arch and ascending aorta is not only necessary for managing chronic aortic disease, but also acute type A dissection. Recently there has been significant attention paid to the fate of the false lumen in type A dissection, including the acute consequences of malperfusion and the late consequences of dilation and rupture. As a result, some have suggested that the entire arch should be replaced using an elephant trunk or frozen elephant trunk technique to facilitate later endovascular repair of the descending thoracic aorta if complications of the chronic dissection flap arise. ${ }^{24}$ Although Dacron replacement of the entire arch provides a robust repair, it comes at the expense of a more complex operation with more risk, including an inability to reconstruct the dissected arch tissue; stroke; and visceral ischemia from prolonged circulatory arrest, bleeding from the distal anastomosis, and coagulopathy associated with deep hypothermia. Hybrid arch techniques have also been advocated for the management of acute type A dissection. ${ }^{6,7,25,26}$ Many of these approaches involve circulatory arrest and intraoperative deployment of an endovascular stent graft in the descending thoracic aorta similar to previously described approaches by Marullo and colleagues ${ }^{10}$ and Diethrich and colleagues. ${ }^{26}$ Our technique involving replacement of the ascending aorta, arch debranching, and antegrade endovascular stent graft deployment without circulatory arrest has the potential to decrease bleeding associated with arch reconstruction under deep hypothermia; to decrease the risk of stroke by eliminating circulatory arrest; to treat visceral malperfusion by opening up the true lumen in the descending aorta; and, in the long-term, may decrease complications by promoting thrombosis of the false lumen.

Although our study is cohort study limited by its small size and relatively short-term follow-up, it suggests that the early results of a type II hybrid arch, single-stage operation are acceptable. It is an approach that can address both acute and chronic diffuse thoracic aortic pathology involving the arch and can be performed with an low rate of perioperative morbidity and mortality and a rate of stent complication and endovascular intervention that is comparable with those in other large hybrid arch and open repair series. ${ }^{1,19}$ Longer-term follow-up will determine if there are significant negative long-term consequences to stent graft placement in the more hostile environment of the arch and ascending aorta with its angulated anatomy, increased motion, and shear stress.

\section{References}

1. Etz CD, Plestis KA, Kari FA, Luehr M, Bodian CA, Spielvogel D, et al. Staged repair of thoracic and thoracoabdominal aortic aneurysms using the elephant trunk technique: a consecutive series of 215 first stage and 120 complete repairs. Eur J Cardiothorac Surg. 2008;34:605-15. 
2. Czerny M, Weigang E, Sodeck G, Schmidli J, Antona C, Gelpi G, et al. Targeting landing zone 0 by total arch rerouting and TEVAR: midterm results of a transcontinental registry. Ann Thorac Surg. 2012;94:84-9.

3. Gelpi G, Vanelli P, Mangini A, Danna P, Contino M, Antona C. Hybrid aortic arch repair procedure: reinforcement of the aorta for a safe and durable landing zone. Eur J Vasc Endovasc Surg. 2010;40:709-14.

4. Geisbusch P, Kotelis D, Muller-Eschner M, Hyhlik-Durr A, Bockler D. Complications after aortic arch hybrid repair. J Vasc Surg. 2011;53:935-41.

5. Kent WDT, Wong JK, Herget EJ, Bavaria JE, Appoo JJ. An alternative approach to diffuse thoracic aortomegaly: on-pump hybrid total arch repair without circulatory arrest. Ann Thorac Surg. 2012;93:326-8.

6. Uchida N, Shibamura H, Katayama A, Shimada N, Sutoh M, Ishihara H. Operative strategy for acute type A aortic dissection: ascending aortic or hemiarch versus total arch replacement with frozen elephant trunk. Ann Thorac Surg. 2009;87:773-7.

7. Jakob H, Tsagakis K. Debakey type I dissection: when hybrid stent-grafting is indicated? J Cardiovasc Surg. 2010;51:633-40.

8. Rampoldi V, Trimarchi S, Eagle KA, Nienaber CA, Oh JK, Bossone E, et al. Simple risk models to predict surgical mortality in acute type A aortic dissection: the International Registry of Acute Aortic Dissection score. Ann Thorac Surg. 2007; 83:55-61.

9. Diethrich EB, Ghazoul M, Wheatley GH, Alpern JB, Rodriquez-Lopez JA, Ramaish VG. Great vessel transposition for antegrade delivery of TAG endoprosthesis in the proximal aortic arch. J Endovasc Ther. 2006;12:583-7.

10. Marullo AGM, Bichi S, Pennetta RA, Di Matteo G, Cricco AM, Specchia L, et al. Hybrid aortic arch debranching with staged endovascular completion in DeBakey type 1 aortic dissection. Ann Thorac Surg. 2010;90:1847-53.

11. Kent WDT, Appoo JJ, Herget EJ, Wong JK. Ascending, total arch and descending thoracic aortic repair for acute Debakey type I aortic dissection without circulatory arrest. Ann Thorac Surg. 2012;94:59-61.

12. Chang Q, Tian C, Wei Y, Qian X, Sun X, Yu C. Hybrid total arch repair without deep hypothermic circulatory arrest for acute type A aortic dissection. J Thorac Cardiovasc Surg. 2013;146:1393-8.

13. Milewski RK, Szeto WY, Pochettino A, Moser GW, Moeller P, Bavaria JE. Have hybrid procedures replaced open aortic arch reconstruction in high-risk patients? A comparative study of elective open arch debranching with endovascular stent graft placement and conventional elective open total and distal aortic arch reconstruction. J Thorac Cardiovasc Surg. 2010;140:590-7.

14. Koullias GJ, Wheatley GH. State-of-the-art hybrid procedures for the aortic arch: a meta-analysis. Ann Thorac Surg. 2010;90:689-97.
15. Matalanis G, Durairaj M, Brooks M. A hybrid technique of aortic arch branch transposition and antegrade stent graft deployment for complete arch repair without cardiopulmonary bypass. Eur J Cardiothorac Surg. 2006;29: 611-2.

16. Kpodonu J, Diethrich EB. Hybrid interventions for the treatment of the complex aortic arch. Perspect Vasc Surg Endovasc Ther. 2007;19:174-84.

17. Shin JH, Yoon HK, Chung CH, Choo SJ, Kim J, Hwang JY, et al. Hybrid procedure with antegrade stent-graft placement for aortic arch aneurysms: preliminary experience in eight patients aortic insufficiency. J Vasc Interv Radiol. 2011;22: $148-54$.

18. Lee CW, Beaver TM, Klodell CT Jr, Hess PJ Jr, Martin TD, Feezor RJ, et al. Arch debranching versus elephant trunk procedures for hybrid repair of thoracic aortic pathologies. Ann Thorac Surg. 2011;91:465-71.

19. Holt PJ, Johnson C, Hinchliffe RJ, Morgan R, Jahingiri M, Loftus IM, et al Outcomes of the endovascular management of aortic arch aneurysm: implications for management of the left subclavian artery. J Vasc Surg. 2010; 51:1329-39.

20. Melissano G, Civilini E, Bertoglio L, Calliari F, Setacci F, Calori G, et al. Results of endografting of the aortic arch in different landing zones. Eur J Vasc Endovasc Surg. 2007;33:561-6.

21. Ueda T, Fleishmann D, Dake M, Rubin GD, Sze DY. Incomplete endograft apposition to the aortic arch: bird-beak configuration increases risk of endoleak formation after thoracic endovascular aortic repair. Radiology. 2010;255:645-52.

22. Eggebrecht H, Thompson M, Rousseau H, Czerny M, Lonn L, Mehta RH, et al Retrograde ascending aortic dissection during or after thoracic aortic stent graft placement: insight from the European registry on endovascular aortic repair complications. Circulation. 2009; 120:S276-81.

23. Di Bartolomeo R, Pacini D, Savini C, Pilato E, Martin-Suarez S, Di Marco L, et al. Complex thoracic aortic disease: single-stage procedure with the frozen elephant trunk technique. J Thorac Cardiovasc Surg. 2010;140:S81-5.

24. Desai ND, Pochettino A. Distal aortic remodeling using endovascular repair in acute Debakey I aortic dissection. Semin Thorac Cardiovasc Surg. 2009;21:387-92.

25. Chen L-W, Wu X-J, Lu L, Zhang G-C, Yang G-F, Yang Z-W, et al. Total arch repair for acute Type A aortic dissection with 2 modified techniques: open single-branched stent graft placement and reinforcement of the dissected arch vessel stump with stent graft. Circulation. 2011;123:2536-41.

26. Diethrich EB, Ghazoul M, Wheatley GH, Alpern J, Rodriguez-Lopez J, Ramaiah V, et al. Surgical correction of ascending Type A thoracic aortic dissection: simultaneous endoluminal exclusion of the arch and distal aorta. J Endovasc Ther. 2005;12:660-6. 